\title{
Forward and Backward Detectable Gray-Scale Data Storage System in Polymeric Material
}

\author{
E. Sungur, D. Carrara, G. Taupier, A. Fort, L. Mager, A. Barsella, and K. D. Dorkenoo
}

Institut de Physique et Chimie des Matériaux de Strasbourg, UMR CNRS, ULP 7504, 23 rue du Loess, B.P. 43, 67034 Strasbourg Cedex 2, France

Correspondence should be addressed to K. D. Dorkenoo, kdorkeno@ipcms.u-strasbg.fr

Received 19 February 2009; Accepted 10 August 2009

Recommended by Lucimara Roman

\begin{abstract}
We describe a fast way to encode a gray-scale image with quadratic properties in polymer thin film doped with azo dye. Under a two photon microscopy setup, we induced disorientation in corona-poled azo dye copolymer thin films by a focused near infrared (IR) femtosecond laser beam of variable exposure time. In situ, the sample was then scan to detect the second harmonic signal. We have also tested the backward detection which can provide reading and writing through a single microscope objective. In addition, we were able to store binary $3 \mathrm{D}$ information in the bulk of a $50 \mu \mathrm{m}$ thick film of the same material.
\end{abstract}

Copyright () 2009 E. Sungur et al. This is an open access article distributed under the Creative Commons Attribution License, which permits unrestricted use, distribution, and reproduction in any medium, provided the original work is properly cited.

\section{Introduction}

Many efforts were put in polymeric material to use them as a fast data recording system $[1,2]$. To cite some examples, holographic recording have been successfully used to encode images in large volumes of poly (methyl methacrylate) (PMMA) [3, 4]. Jia et al. have presented a generation of continuous gray levels in three-dimensional diffractive optical elements and have demonstrated the use of the two-photon polymerization method for fabricating threedimensional diffractive optical elements of continuous gray levels [5]. Li et al. have reported a rewritable polarizationencoded multilayer data storage method with a polymer film doped with the azo dye DMNPAA (2,5-dimethyl-4-(pnitrophenylazo) anisole) under two-photon excitation by a linearly polarized femtosecond laser beam at $780 \mathrm{~nm}$, where the optical axis of DMNPAA molecules can be oriented perpendicular to the direction of the beam via a transcis-trans isomerization process [6]. In this work, we have improved our approach to encode information in DR1grafted PMMA [7-9]. In particular, we have investigated the possibility to encode information in $3 \mathrm{D}$ using the quadratic response of a polymer film. As in our previous work [79], the approach consists in using the optically induced disorientation of the DR1 molecules. We started from a film where the push-pull azo-chromophores have been oriented and then frozen in a given orientation through conventional poling techniques. This provides us with a sample which uniformly emits a second harmonic signal (all pixels set to white). The two-photon absorption (TPA) process is then used to locally disorient the chromophores in desired microscopic volumes via cis-trans isomerization cycles. The resulting decrease in second harmonic intensity is directly related to the amount of induced disorientation. While in our previous work we used a spectrometer to follow the evolution of the signal and for data readout, we have now replaced it with a photomultiplier, which provides a faster and simpler data acquisition.

Our samples were prepared from a commercially purchased poly-methyl-methacrylate (PMMA) copolymer, where one monomer out of ten carries a Dispers Red (DR1) molecule (Specific Polymer, molecular weight = $20000 \mathrm{~g} / \mathrm{mol}, \mathrm{Tg}=125^{\circ} \mathrm{C}$ ). To prepare a thin film, the solution of DR1 functionalyzed PMMA in 1,1,2-trichloroethan is spin coated on a transparent microscope slide and left to dry at $80^{\circ} \mathrm{C}$ for 1 hour yielding films with a typical thickness of $400 \mathrm{~nm}$ as measured by a DekTak Profilometer. In the case of thick $(50 \mu \mathrm{m})$ films, $6 \%$ weight of DR1 doped PMMA was dissolved in chloroform. The solution was filtered through a $0.45 \mu \mathrm{m}$ Teflon filter and cast directly into a stainless steel ring 


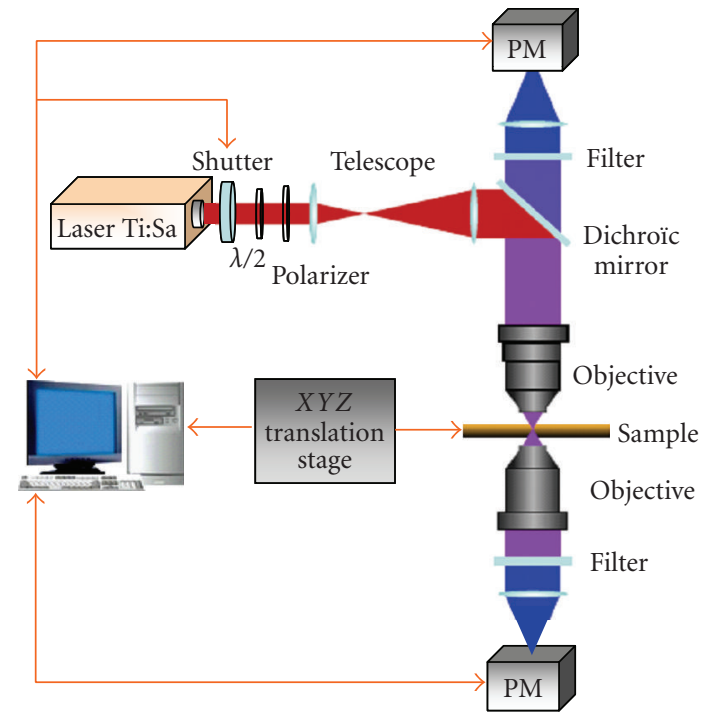

FIgURE 1: Schematic diagram of the experimental setup. The two filters are used to detect only the SHG signal in the forward and backward directions.

on a leveled mirror. The initial evaporation was performed in a glove bag in a solvent-enriched atmosphere. After 24 hours, the film was removed from the mirror plate by immersion in water. The film was further dried under vacuum at $100^{\circ} \mathrm{C}$ to remove the residual solvent. Our film (thin or thick) was then subjected to elevated temperature corona poling on a heating plate at $130^{\circ} \mathrm{C}$ in a normal atmosphere. This procedure yields samples where the chromophores are sufficiently oriented on average to generate a second harmonic generation (SHG) signal which is nearly uniform over areas of about $2 \times 2 \mathrm{~cm}^{2}$.

The experimental setup is presented in Figure 1. Reading and writing are performed by placing the sample between two identical microscope objectives $(\times 20)$ : the first is used to provide the laser beam, the second to collect the SHG signal. The sample is held by a $3 \mathrm{D}$ translation system which allows us to scan its position along the three $x, y, z$ axes with a resolution of $200 \mathrm{~nm}$. The laser source is a Tsunami Ti:saphire tunable laser $(670-1100 \mathrm{~nm})$ with $120 \mathrm{fs}$ pulse duration and $80 \mathrm{MHz}$ repetition rate. Two different power levels are used for reading $(\sim 20 \mathrm{~mW})$ and writing $(\sim 70 \mathrm{~mW})$, obtained by adjusting a half-wave plate and a Glan-Taylor polarizer. The low intensity of the reading case is chosen to avoid further isomerization.

\section{Calibration of the System}

In order to control the level of disorientation of the chromophores and, consequently, to obtain the desired amplitude of the SHG signals, we have induced local TPA processes on successive areas of the sample by exposing them to increasing levels of irradiation. The written areas have then been read back to determine the SHG intensities of the different zones. We have started by calculating the maximum theoretical number of levels that can be recorded in the structure. We measured the average intensity and standard deviation of a poled (white) area and of a completely disoriented (black) area. At $70 \mathrm{~mW}$ laser power, the exposure time for a completely black area is around 5 seconds. The photon counts for these two areas are $12700 \pm 380$ and $130 \pm 40$. If we want two gray levels to be separated by at least two standard deviations, and assume the worst case of $\sigma=380$, this would allow for 16 levels to be written in the material. In reality, we cannot use the entire range, as long-time exposure results not only in chromophore disorientation but also in the degradation of the polymer matrix. We have then one additional constraint which limits the maximum exposure time. White-light imaging of the sample indicates that for exposure times higher than 1 second, material degradation becomes relevant. Figure 2(a) shows a photon count measurement together with a white-light image of the sample: only the long-exposure square is visible (5 seconds), while the other two ( 0.4 second and 1.3 seconds) do not result in sample degradation. The lowest count available is then raised from 130 to around 2200, which corresponds to the darkest area with no material damage (exposure time of 1.3 seconds at $70 \mathrm{~mW}$ ). The number of usable gray levels is then reduced from 16 to 13 . Our tests indicate that this number is optimistic, since inhomogeneities in the sample can increase the deviation of the SHG signal from the expected value. Figure 2(c) shows the test grayscale image that we wrote in the sample. The exposure times go from 0.05 to 0.85 second, split in 5 gray levels ( 6 levels in total, if including the unexposed areas). Note that since the SHG intensity is not a linear function of the exposure time, the exposure times for the gray levels do not increase linearly, but follow roughly an extended exponential curve $[8,10]$.

\section{Thin Film Recording}

In order to demonstrate the capability to record an image, we have taken a bitmap of a popular comic's hero and quantified it on 5 levels, then we have proceeded to write it pixel-bypixel. The gray levels used correspond to exposure times of $0,0.02,0.12,0.37$, and 1 second, which are determined to reproduce the luminance of the gray levels used in the original image, for a writing power of $70 \mathrm{~mW}$. The recorded area is $200 \mu \mathrm{m}$ by $300 \mu \mathrm{m}$. Figure 3 shows the original image as well as the SHG read back from the written area. For comparison, the SHG signal has been renormalized to cover the same luminosity interval than the original image, the darkest area being normalized to black while the clearest area to white. While the overall quality of the image is very good (Figure 3(b)), we can see the "smoothing out" due to the finite spatial resolution of the writing and reading process as well as some deviation from the original colors. Some of them are due to irregularities of the sample, like the dark dot in the lower-right corner or the white spots on the character's nose. Another source of error are inhomogeneities in the material, which result in a global shift of the gray levels, as it appears on the part above the left eye, which is black in the original area, but is read back as dark gray in the reconstruction. Figure 3(c) demonstrates the durability of the sample: it represents the read back of the same area of Figure 3(b) but seven months later, during this time the 


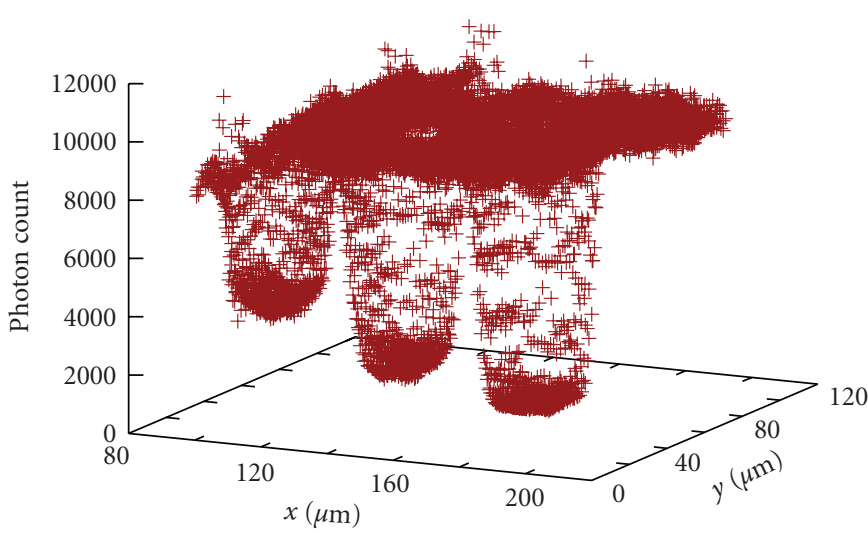

(a)

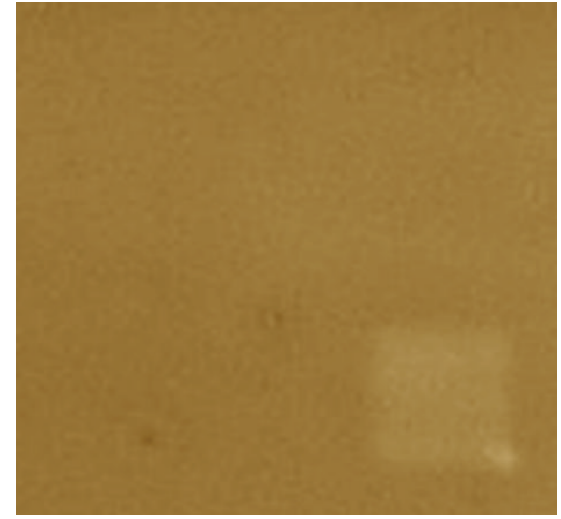

(b)

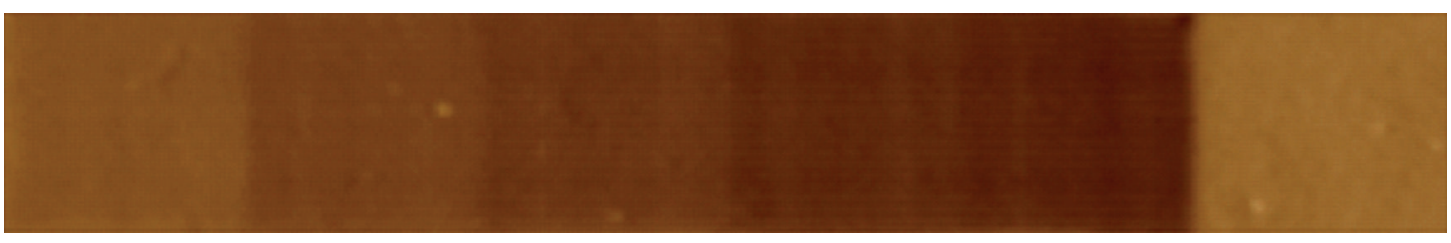

(c)

FIgURE 2: Calibration of the system: (a) photon counts as a function of position for different exposure times: 0.4 second, 1.3 seconds, 5 seconds, (b) white-light image of the same area as (a), showing material degradation for the darkest (lowest count) square, (c) SHG image of 6-level gray scale obtained by varying the exposure time from 0 to 0.85 second. In all cases the laser power is fixed to $70 \mathrm{~mW}$ for writing and the readout time is $20 \mathrm{~ms} /$ pixel at $20 \mathrm{~mW}$.

sample has been kept in the dark and at room temperature. We can notice the noisy aspect of the image, which is due to the decrease of the SHG signal resulting from the aging process and a worse signal/noise ratio. Nevertheless the image of Asterix is well preserved and the grey levels are still distinguishable.

We have also performed another test to investigate the possibility of reading and writing through a single microscope objective, an approach which would simplify the read/write system. We observed that backward-detection provides us with enough signal. Two possible sources of this signal are the back-reflection of the SHG beam at the bottom sample/air interface or directly the backward propagating SHG (back-SHG). In the case where second harmonic emitting molecules are tightly clustered around the focal point of the fundamental beam (small size of the zone of possible SHG emission compared to the SHG active volume size), taking into account momentum conservation arguments and phase anomalies at that focal center, the SHG beam can propagate in the backward direction [1113]. The use of an index-matching fluid to eliminate backreflection from the sample/air interface has shown that around $50 \%$ of the signal comes from back-SHG. The backreflected SHG beam does not have the same geometry as the incoming beam, but since we do not use a pinhole in front of the photomultiplier, even if the spot is not correctly focused, the area of the photomultiplier can collect it. Compared to a "classical" confocal setup, here we are relying completely on the spatial selectivity of the excitation to provide the $3 \mathrm{D}$ resolution, and on the narrow pass-band filter to keep only the SHG component and eliminate any fluorescence.

Figure 4(a) shows the evolution of the SHG signals intensities as a function of the wavelength for the forward and the backward components. As expected, when approaching resonance both signals increase, the back-collected one showing a reduced increase. A possible explanation can be the fact that the part of the back-collected SHG, which comes from the reflection at the bottom interface, must cross again the film, which will introduce additional absorption. We have also tested this on a black/white image, Figures 4(b) and 4 (c) show the two read back images obtained from the two photomultipliers.

\section{Thick Film Recording}

In the recording test of the thick $(50 \mu \mathrm{m})$ film we have chosen to write an image close to the lower surface of the film. Figure 5 shows a picture of a thick sample. The writing and reading process are identical to the one of the thin film.

The main problem is that the homogeneity of the sample is not as good as in the case of a thin film, even on planes which are unaffected by the writing process, the signal fluctuates too much to obtain anything more than 2-level writing. For example, the reading in the bulk of the thick sample giving an average signal of 150 photons, presents some peak signals of 1000 photons. We have then limited ourselves to a binary image. At the lowest plane $z=0 \mu \mathrm{m}$ we draw a circle adjacent to a disc and then we continued to draw 


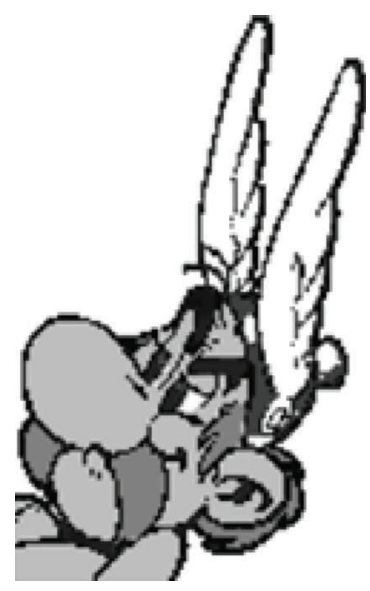

(a)

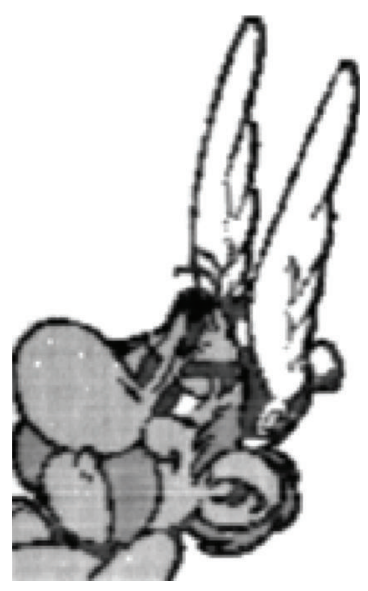

(b)

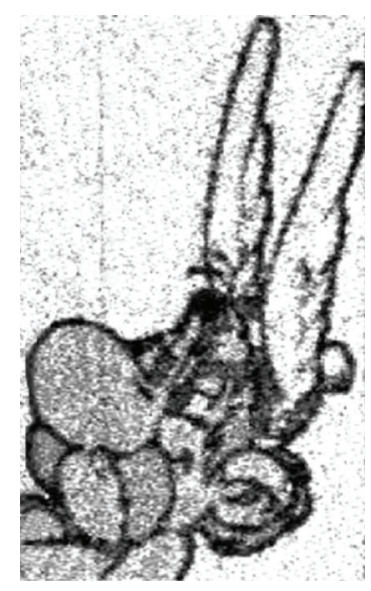

(c)

FIGURE 3: Asterix image: (a) the original grayscale image, quantized on 5 gray levels, (b) the read back from the sample $($ size $200 \times 300 \mu \mathrm{m})$, rescaled to cover the entire black to white range and quantized on 5 gray levels to allow direct comparison with the original image, (c) the read back of the same image 7 months later. In each case the readout time is $20 \mathrm{~ms} /$ pixel at a power of $20 \mathrm{~mW}$.

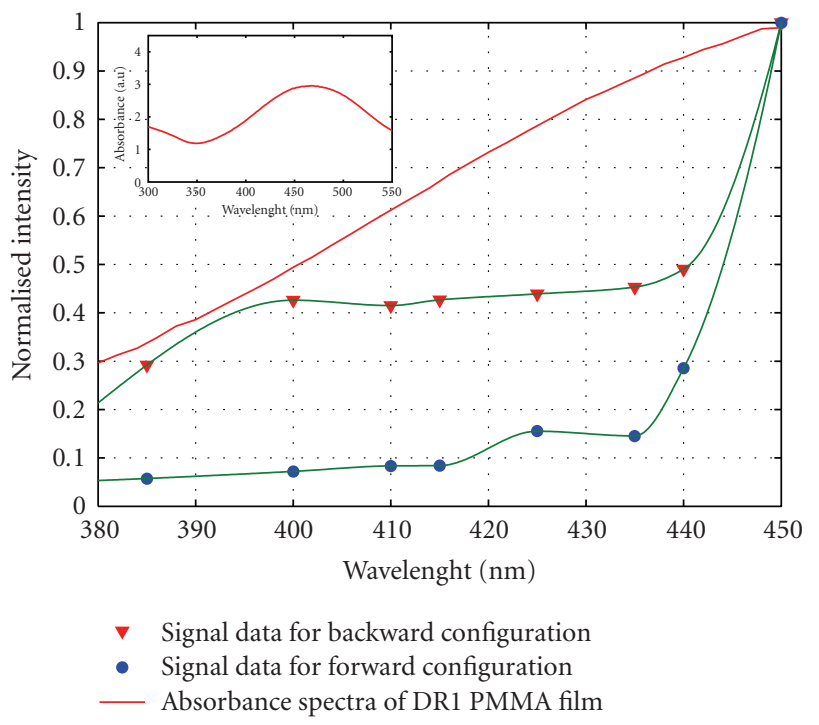

(a)

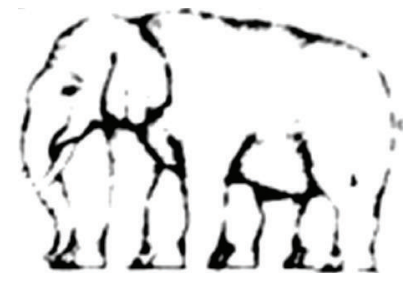

(b)

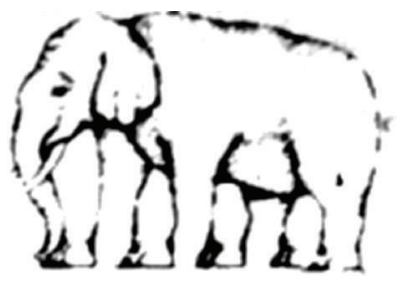

(c)
FIGURE 4: (a) Evolution of the forward and backward SHG intensities as a function of wavelength, showing the increase of signal as the wavelength approaches the DR1 resonance. The inset represents the absorbance spectra of DR1. (b) and (c) Reconstructed and renormalized B/W image from respectively the forward and backward SHG signals.

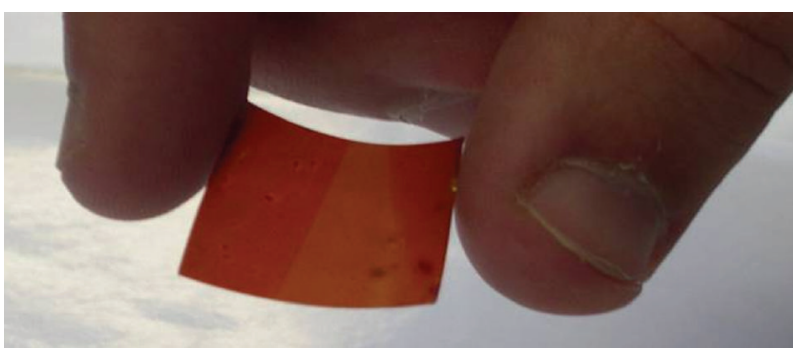

FIGURE 5: Thick film sample.

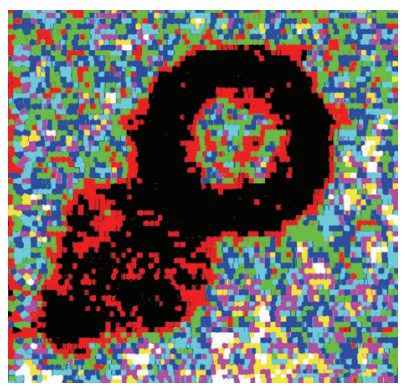

(a)

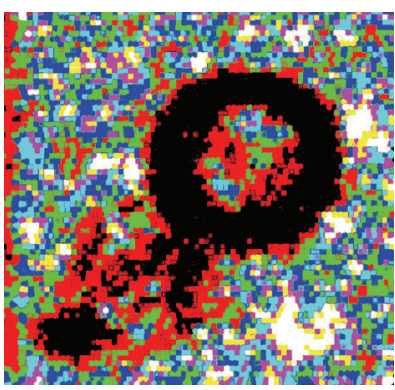

(b)
Figure 6: SHG signal intensity measured on two separate depth planes (a) $z=0 \mu \mathrm{m}$ and (b) $z=20 \mu \mathrm{m}$.

the circle alone in upper planes separated by $2 \mu \mathrm{m}$. We have then performed readings at different planes. Figure 6 shows the SHG image for the planes $z=0 \mu \mathrm{m}$ and $z=20 \mu \mathrm{m}$. We can see that the disc has already disappeared from the upper image. The noisy aspect of Figure 6 shows that the quality of the image lower than the case of a thin film, but it is clear that the information is detectable in the bulk of the material. 


\section{Conclusion}

We have presented an approach to encode information in polymeric materials functionalized with azo-dye molecules based on the disorientation of the azo-chromophores by successive isomerization cycles induced through two-photon absorption processes and resulting in a decrease of the SHG signal. The ability to control the disorientation rates allows the encoding of grey scale images. In this study we have determined the relevant grey scale level number that we can use in this material and presented an example of a high quality image. This encoding method can also be used for $3 \mathrm{D}$ storage. As an example we stored a binary image in the bulk of the material but the low quality of thick samples is currently a limiting factor, nevertheless the technique seems to be a promising tool and effort should be made to improve the material quality.

\section{Acknowledgment}

Authors wish to thank Institut TELECOM for financial support through its "Future et ruptures" programme.

\section{References}

[1] D. A. Parthenopoulos and P. M. Rentzepis, "Three-dimensional optical storage memory," Science, vol. 245, no. 4920, pp. 843-845, 1989.

[2] M. Maeda, H. Ishitobi, Z. Sekkat, and S. Kawata, "Polarization storage by nonlinear orientational hole burning in azo dyecontaining polymer films," Applied Physics Letters, vol. 85, pp. 351-353, 2004.

[3] G. J. Steckman, I. Solomatine, G. Zhou, and D. Psaltis, "Characterization of phenanthrenequinone-doped poly(methyl methacrylate) for holographic memory," Optics Letters, vol. 23, pp. 1310-1312, 1998.

[4] S. H. Lin, K. Y. Hsu, W. Z. Chen, and W. T. Whang, "Phenanthrenequinone-doped poly(methyl methacrylate) photopolymer bulk for volume holographic data storage," Optics Letters, vol. 25, pp. 451-453, 2000.

[5] B. Jia, J. Serbin, H. Kim, B. Lee, J. Li, and M. Gu, "Use of twophoton polymerization for continuous gray-level encoding of diffractive optical elements," Applied Physics Letters, vol. 90, Article ID 073503, 2007.

[6] X. Li, J. W. Chon, S. Wu, R. A. Evans, and M. Gu, "Rewritable polarization-encoded multilayer data storage in 2,5-dimethyl4-(p-nitrophenylazo)anisole doped polymer," Optics Letters, vol. 32, pp. 277-279, 2006.

[7] D. Gindre, A. Boeglin, A. Fort, L. Mager, and K. D. Dorkenoo, "Rewritable optical data storage in azobenzene copolymers," Optics Express, vol. 14, no. 21, pp. 9896-9901, 2006.

[8] D. Gindre, A. Boeglin, G. Taupier, et al., "Toward submicrometer optical storagethrough controlled molecular disordervin azo-dye copolymer films," Journal of Optical Society of America B, vol. 24, pp. 532-537, 2007.

[9] D. Gindre, I. Ka, A. Boeglin, A. Fort, and K. D. Dorkenoo, "Image storage through gray-scale encoding of second harmonic signals in azo-dye copolymers," Applied Physics Letters, vol. 90, Article ID 094103, 2007.

[10] G. William and D. C. Watt, "Non-symetrical dielectric relaxation behaviour arising from a simple empirical decay function," Transactions Faraday Society, vol. 66, pp. 80-85, 1970.

[11] S. E. Harris, "Proposed backward wave oscillation in the infrared," Applied Physics Letters, vol. 9, no. 3, pp. 114-116, 1966.

[12] R. M. Williams, W. R. Zipfel, and W. W. Webb, "Interpreting second-harmonic generation images of collagen I fibrils," Biophysical Journal, vol. 88, no. 2, pp. 1377-1386, 2005.

[13] L. Moreaux, O. Sandre, and J. Mertz, "Membrane imaging by second-harmonic generation microscopy," Journal of the Optical Society of America B, vol. 17, pp. 1685-1694, 2000. 

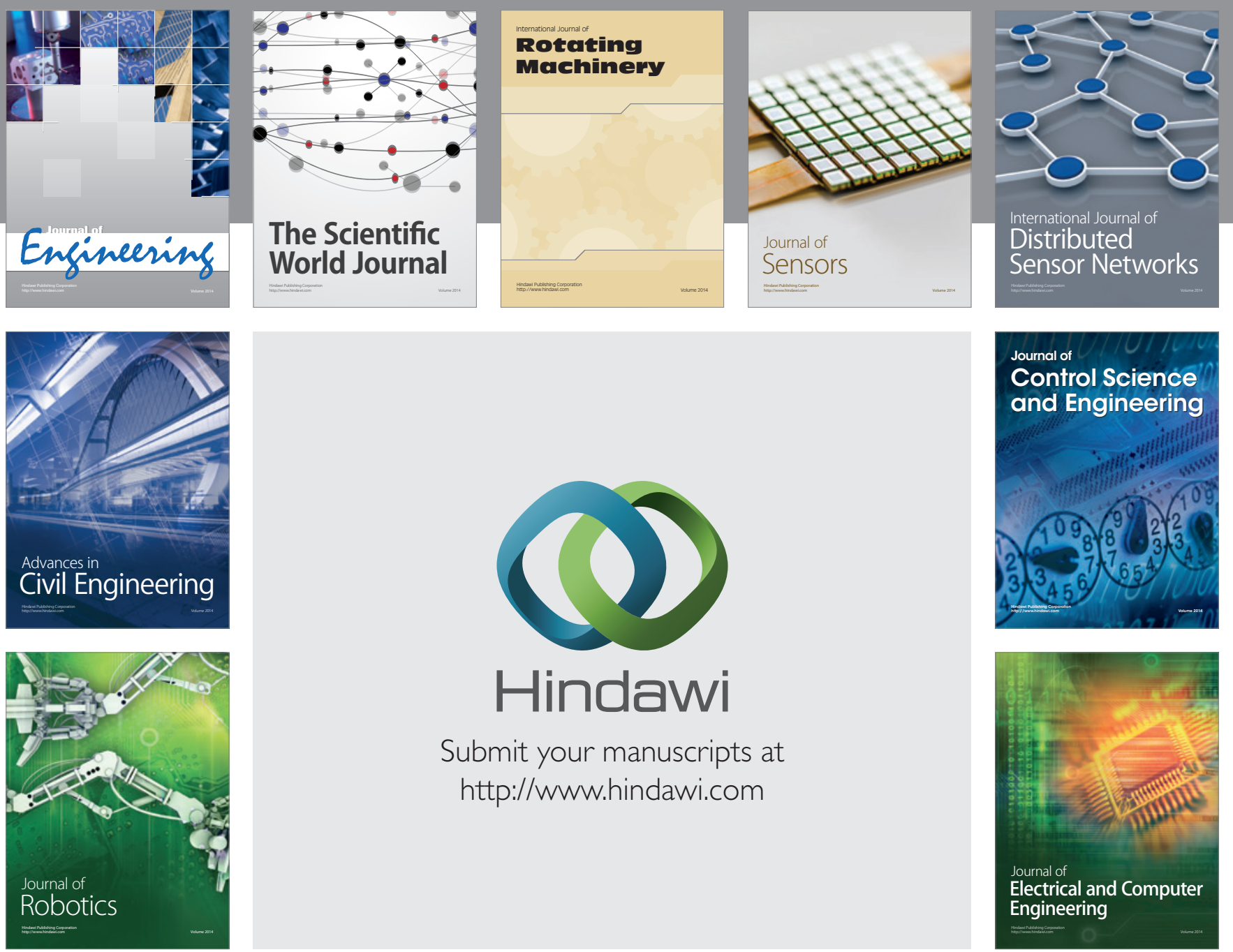

Submit your manuscripts at

http://www.hindawi.com
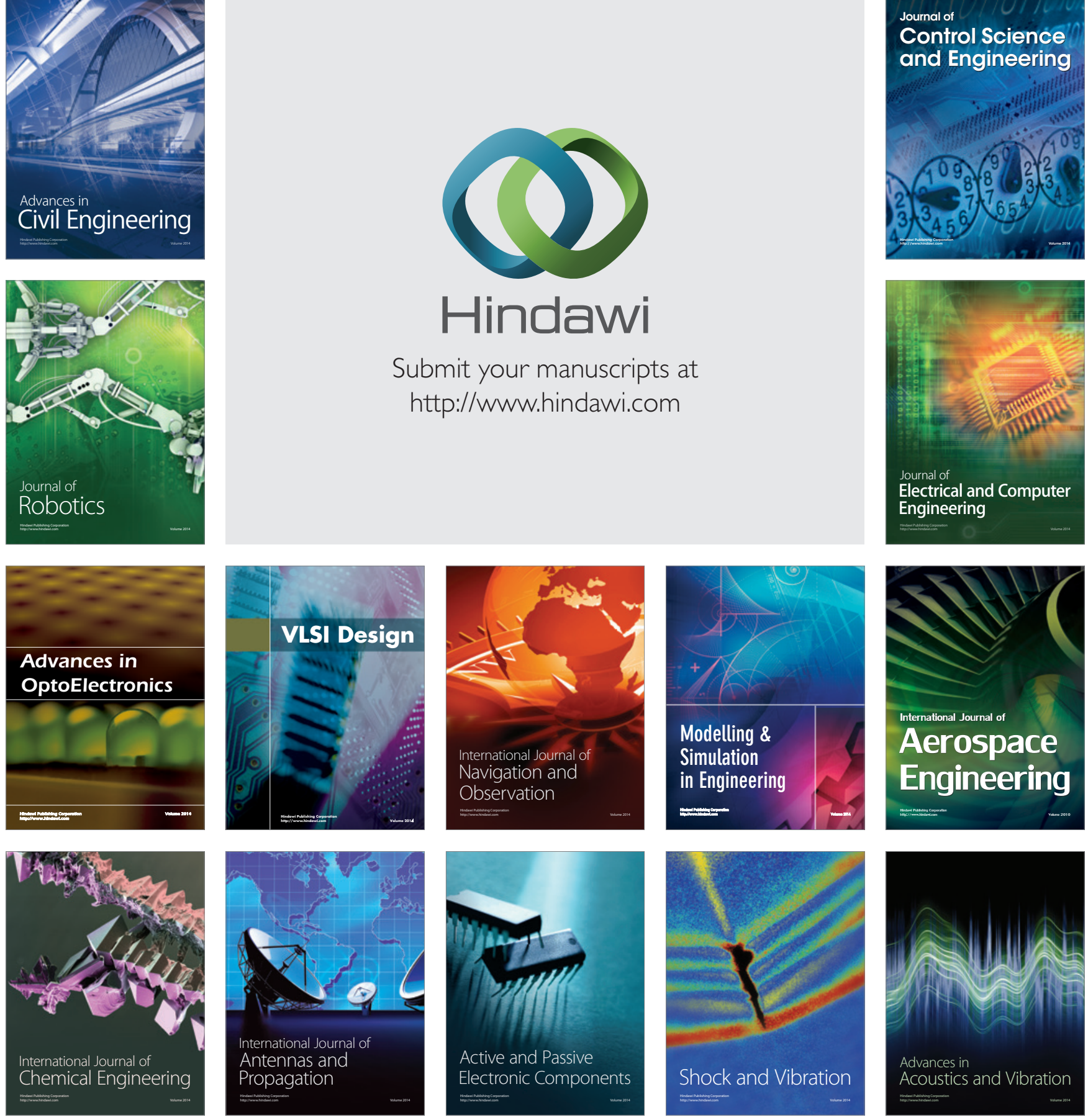